\title{
Role of multistability in the transition to chaotic phase synchronization
}

\author{
Postnov, D.E.; Vadivasova, T.E.; Sosnovtseva, Olga; Balanov, A.G.; Anishchenko, V.S.; Mosekilde, Erik
}

\section{Published in:}

Chaos

Link to article, DOI:

10.1063/1.166394

Publication date:

1999

Document Version

Publisher's PDF, also known as Version of record

Link back to DTU Orbit

Citation (APA):

Postnov, D. E., Vadivasova, T. E., Sosnovtseva, O., Balanov, A. G., Anishchenko, V. S., \& Mosekilde, E. (1999). Role of multistability in the transition to chaotic phase synchronization. Chaos, 9(1), 227-232.

https://doi.org/10.1063/1.166394

\section{General rights}

Copyright and moral rights for the publications made accessible in the public portal are retained by the authors and/or other copyright owners and it is a condition of accessing publications that users recognise and abide by the legal requirements associated with these rights.

- Users may download and print one copy of any publication from the public portal for the purpose of private study or research.

- You may not further distribute the material or use it for any profit-making activity or commercial gain

- You may freely distribute the URL identifying the publication in the public portal 


\title{
Role of multistability in the transition to chaotic phase synchronization
}

\author{
D. E. Postnov, T. E. Vadivasova, O. V. Sosnovtseva, A. G. Balanov, \\ and V. S. Anishchenko \\ Department of Physics, Saratov State University, Astrakhanskaya Street 83, Saratov, 410026, Russia \\ E. Mosekilde ${ }^{a}$ \\ Department of Physics, The Technical University of Denmark, 2800 Lyngby, Denmark
}

(Received 19 May 1998; accepted for publication 22 September 1998)

\begin{abstract}
In this paper we describe the transition to phase synchronization for systems of coupled nonlinear oscillators that individually follow the Feigenbaum route to chaos. A nested structure of phase synchronized regions of different attractor families is observed. With this structure, the transition to nonsynchronous behavior is determined by the loss of stability for the most stable synchronous mode. It is shown that the appearance of hyperchaos and the transition from lag synchronization to phase synchronization are related to the merging of chaotic attractors from different families. Numerical examples using Rössler systems and model maps are given. (C) 1999 American Institute of Physics. [S1054-1500(99)00201-3]
\end{abstract}

The interaction between two or more chaotic oscillators can produce a number of different synchronization phenomena, depending on the degree to which the oscillators adjust their motion in accordance with one another. A variety of technical applications of such synchronization phenomena are presently being considered, e.g., for secure communication and for the surveillance and control of systems that operate in a chaotic regime. Chaotic synchronization may also play an essential role for the regulation of many biological systems where an individual functional unit displays complex dynamics. In the present paper we investigate the bifurcations that can occur when chaotic synchronization is established between two period-doubling systems with different basic periodicities. We show how this synchronization gives rise to a nested structure of phase synchronized regions.

\section{INTRODUCTION}

Chaotic synchronization is a topic of fundamental interest in many areas of science (Refs. 1-6, and references cited therein). It has also been suggested that chaotic synchronization can be applied in connection, for instance, with the development of new methods for secure communication and for the monitoring of dynamical systems. ${ }^{4-6}$ A basic question in this area of research is the following: Given a system of interacting, slightly nonidentical chaotic oscillators with a certain distribution of their natural frequencies, to what degree will the oscillators adjust their motion in accordance with one another so as to attain some kind of overall coherence? Several aspects of this problem have been considered in the literature, with full synchronization, ${ }^{7}$ generalized synchronization, ${ }^{8,9}$ and phase synchronization, ${ }^{10,11}$ represent-

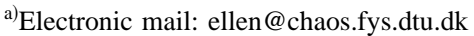

ing different forms of the same basic phenomenon. However, the transitions to and between these different types of synchronization are not understood in detail.

It is well-known that transitions from regular to chaotic oscillations demonstrate various forms of scaling and universality properties. ${ }^{12}$ Transitions within chaos are associated with complex phenomena such as crises, ${ }^{13}$ symmetrybreaking bifurcations, ${ }^{14}$ attractor bubbling, and local (or global) riddling phenomena, ${ }^{15}$ as well as chaos-chaos and chaos-hyperchaos intermittency. ${ }^{16-18}$ Recently, there has been a growing interest in a bifurcational interpretation of the phenomena that take place at the boundary of chaotic synchronization. Anishchenko et al. ${ }^{3}$ have associated this boundary with an accumulation of curves of tangent bifurcations of saddle cycles, and a more recent study by Pikovsky et $a l .{ }^{19}$ suggests that attractor-repeller collisions take place at the transition to chaotic synchronization, thus drawing on the analogy with the tangent bifurcation of a limit cycle. Most recently, ${ }^{20}$ the transition to phase synchronization was described as a boundary crisis mediated by unstableunstable pair bifurcations on a branched manifold.

Multistability, i.e., the coexistence of a set of attractors in the phase space of a dynamical system, is another typical phenomenon for nonlinear systems. The development of different families of regular and chaotic attractors for coupled oscillators has been investigated by several authors. ${ }^{21-23}$ As shown by Astakhov et al., ${ }^{21}$ for instance, two dissipatively coupled, identical oscillators following the period-doubling route to chaos will exhibit a hierarchy of bifurcations in which different families of attractors emerge. For two coupled Rössler systems, Rasmussen et al. ${ }^{22}$ have found the replacement of some of the period-doubling bifurcations by torus bifurcations leading to quasiperiodicity, frequencylocking, and the emergence of new nonsymmetric families of attractors. Anishchenko et ll $^{23}$ have shown that this multistability is structurally stable with respect to a mismatch between the basic frequencies.

In the present paper we study the structure of the syn- 
chronization region for interacting oscillators whose spectrum contains subharmonics $f / 2^{k}(k=1,2, \ldots)$ of the basic frequency. The paper is organized as follows. In Sec. II we introduce two coupled Rössler systems and give survey of the behavior observed in this model. Further, in Sec. III we reduce the problem to a simple mapping. We start with formulas that emulate multistable behavior to guide the search for such phenomena in phase synchronized chaos. Based on the analysis of a one-dimensional map modeling interacting period-doubling systems and on results from numerical simulations of coupled Rössler systems with a mismatch between the basic frequencies, we investigate the structure of the boundary of the synchronization region. We summarize our results in Sec. IV.

\section{DYNAMICS OF COUPLED RÖSSLER SYSTEMS}

As an illustrative example let us consider the wellknown pair of coupled Rössler systems as described by Rosenblum et al.: $:^{10}$

$$
\begin{aligned}
& \dot{x}_{1}=-\omega_{1} y_{1}-z_{1}+c\left(x_{2}-x_{1}\right), \\
& \dot{y}_{1}=\omega_{1} x_{1}+\alpha y_{1}, \\
& \dot{z}_{1}=\beta+z_{1}\left(x_{1}-\mu\right), \\
& \dot{x}_{2}=-\omega_{2} y_{2}-z_{2}+c\left(x_{1}-x_{2}\right), \\
& \dot{y}_{2}=\omega_{2} x_{2}+\alpha y_{2}, \\
& \dot{z}_{2}=\beta+z_{2}\left(x_{2}-\mu\right),
\end{aligned}
$$

where the parameters $\alpha, \beta$, and $\mu$ govern the dynamics of each subsystem. $c$ is the coupling parameter, $\omega_{1}=\omega_{0}+\Delta$ and $\omega_{2}=\omega_{0}-\Delta$ are the basic frequencies (we suppose $\left.\omega_{0}=1\right)$, and $\Delta$ is the mismatch between the basic frequencies.

Because synchronization between the two systems involves phase relations it is useful to rewrite (1) in terms of phases and amplitudes. Instant amplitudes $A_{1,2}$ and phases $\Phi_{1,2}$ are introduced by the substitution of variables:

$$
\begin{aligned}
& x_{1,2}=A_{1,2} \cos \Phi_{1,2}, \\
& y_{1,2}=A_{1,2} \sin \Phi_{1,2} .
\end{aligned}
$$

This allows us to recast (1) into the form

$$
\begin{aligned}
\dot{A}_{1,2}= & \alpha A_{1,2}+\left(c A_{2,1} \cos \Phi_{2,1}-A_{1,2}(\alpha+c) \cos \Phi_{1,2}\right. \\
& \left.-z_{1,2}\right) \cos \Phi_{1,2}, \\
\Phi_{1,2}= & \omega_{1,2}-\left(c A_{2,1} / A_{1,2} \cos \Phi_{2,1}-(\alpha+c) \cos \Phi_{1,2}\right. \\
& \left.-z_{1,2} / A_{1,2}\right) \sin \Phi_{1,2}, \\
\dot{z}_{1,2}= & \beta+z_{1,2}\left(A_{1,2} \cos \Phi_{1,2}-\mu\right) .
\end{aligned}
$$

As before, $\mu$ controls the bifurcations of the individual Rössler systems, and $\Delta$ determines the detuning between the two interacting systems. Hence, these two aspects can be separately considered. By numerically integrating (3), it is easy to find $\delta \Phi(t)=\Phi_{2}(t)-\Phi_{1}(t)$ for any oscillating solution.

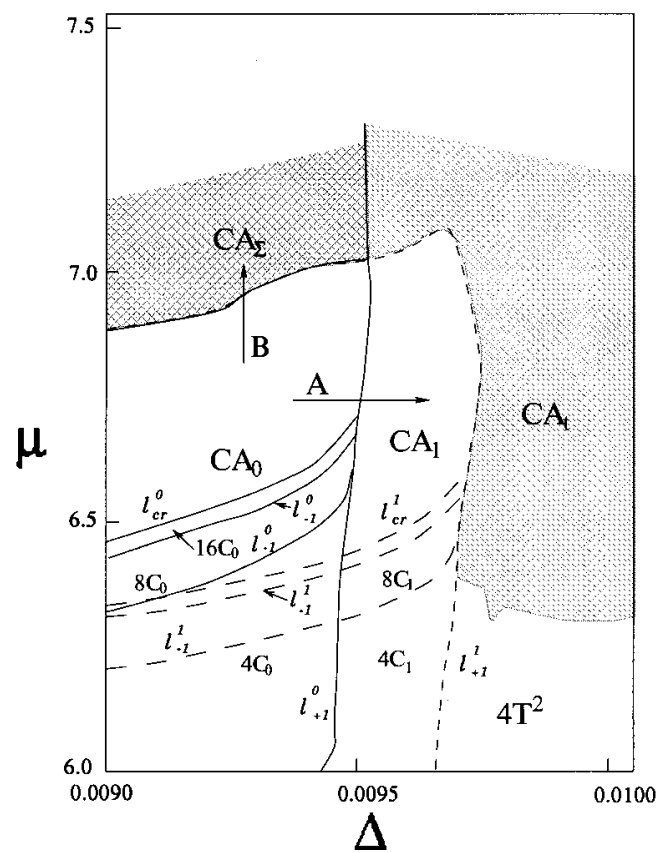

FIG. 1. Bifurcation diagram for two coupled Rössler systems $(\alpha=0.15$, $\beta=0.2, c=0.02)$. The solid curves are bifurcation lines of "in-phase", attractors. The dashed curves show bifurcations of "out-of-phase" attractors.

With $\mu$ and $\Delta$ as active parameters we now perform a detailed bifurcation analysis of the coupled Rössler systems and follow the periodic and chaotic trajectories of different attractor families. For each such family, a phase-locking region appears.

Figure 1 shows a segment of the bifurcation diagram for a synchronous solution on the $(\Delta, \mu)$ parameter plane, while $c, \alpha$, and $\beta$ are fixed. Because the number of synchronous regimes depends on the period of oscillations, new synchronous regimes appear above each line of period-doubling and there exists an infinite but denumerable set of such regimes. Hence, it is difficult to analyze and display all of them in the diagram. Let us consider only attractors from two families: "in-phase" attractors when the phase difference of $x_{1}(t)$ and $x_{2}(t)$ is zero at $\Delta=0$, and "out-of-phase" attractors when the phase difference is $2 \pi$ at $\Delta=0$. These families have the largest basins of attraction. Denote the attractors as $2^{i} C_{0}$ and $2^{i} C_{1}$, respectively, where $i=1,2,3, \ldots$, and $2^{i}$ is the cycle period, normalized in terms of the period of cycle $C_{0}$.

For bifurcational curves of $2^{i} C_{0,1}$ cycles we use the following denotation: $l_{-1}$ is a curve of period-doubling bifurcation of cycles $2^{i} C_{0,1} ; l_{+1}$ is a curve of tangent bifurcation of cycles $2^{i} C_{0,1} ; l_{c r}^{0}$ is a critical curve, corresponding to the accumulation of period-doubling bifurcations of $2^{i} C_{0}$ cycles; and $l_{c r}^{1}$ is a critical curve of family $2^{i} C_{1}$.

Above the critical curves there exists a set of chaotic attractors $2^{i} C A_{0}$ and $2^{i} C A_{1}$. Band-merging bifurcations of these attractors take place. We omit the corresponding bifurcation curves and denote the regions of chaos as $C A_{0}$ and $C A_{1} . C A_{\Sigma}$ appears from a merging of $C A_{0}$ and $C A_{1}$. Investigations have shown that cycles $2^{i} C_{1}$ are stable longer when detuning is applied. Outside the synchronization region, quasiperiodic oscillations $4 T^{2}$ and nonsynchronous chaos $C A_{t}$ are found. 


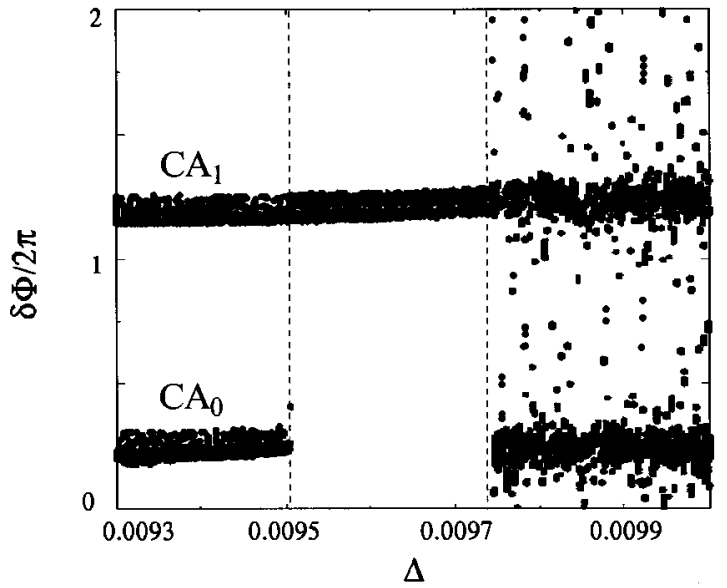

FIG. 2. One-parameter bifurcation diagram for two coupled Rössler systems $(\alpha=0.15, \beta=0.2, c=0.02, \mu=6.7)$.

The coexistence of a set of attractors characterized by different phase shifts is a universal phenomenon for coupled systems with period-doublings, and their main features are often independent of particular properties of a model.

The number of coexisting attractors inside a synchronization region for weak coupling tends to infinity close to the threshold of chaos. When the detuning parameter $\Delta$ is increased, the synchronous chaotic regimes sequentially lose their stability (direction $A$ in Fig. 1). We can construct a bifurcation diagram (Fig. 2) for the different families of chaotic attractors $C A_{0}$ and $C A_{1}$. It is easy to see that the number of possible synchronous chaotic solutions decreases at $\Delta=0.0097$. Moreover, the chaotic attractor $C A_{1}$ is stable in a wider range of detuning parameters and its bifurcation curve forms the boundary of the synchronization region.

Along the direction $B$ (Fig. 1) the number of possible synchronous solutions also decreases, but in a rather different way. As $\mu$ is increased within the chaotic region, a sequence of crises of chaotic attractors takes place. Each crisis reduces the number of possible synchronous regimes by two. Finally, a single chaotic attractor is formed by the merging of chaotic trajectories of all families. The last phenomenon leads to new properties of the chaotic solution and can be diagnosed in different ways.

Let us consider the distribution of phase differences $p$ for chaotic attractors of the various families. Figure 3(a) shows the corresponding plots for the coexisting chaotic attractors $C A_{0}$ and $C A_{1}$ (curves 1 and 2, respectively) and for the attractor that arises as a result of the merging of these attractors (curve 3). It is easy to see that the phase structure of the resulting chaos is rather different from the structure of the chaotic regimes before merging. The observed Gaussianlike phase distribution can not be found for chaos which appears via the period-doubling scenario.

Further investigation shows that the merging of chaotic attractors leads to hyperchaos. Each attractor $\left(C A_{0}\right.$ or $\left.C A_{1}\right)$ is characterized by only one positive Lyapunov exponent. But when the transition to merged chaos occurs, a second direction of exponential instability and, hence, a second positive Lyapunov exponent appear. Figure 3(b) shows the two largest Lyapunov exponents for the attractors of two different families as a function of the parameter $\mu$. The dark points corresponds to $C A_{0}$, and the open circles to $C A_{1}$. For $\Delta=0.0093$ at $\mu=6.97$, a crisis of $C A_{0}$ and $C A_{1}$ takes place that leads to the appearance of a new chaotic attractor $C A_{\Sigma}$. The attractor $C A_{\Sigma}$ contains the trajectories of $C A_{0}$ and $C A_{1}$ and is characterized by two positive Lyapunov exponents. Hence, the emergence of hyperchaos is observed.

Increasing of the coupling coefficient leads to the destruction of multistability because the "out-of-phase" solutions die out. ${ }^{21,22}$ Thus, when coupling is stronger, there exist only attractors corresponding to the zero difference of the phase for the partial oscillations (i.e., $2^{i} C_{0}, 2^{i} C A_{0}$ ). Following the variation of the Lyapunov exponents when the coupling is increased, it is easy to find the transition from hyperchaos to chaos $C A_{0}$ with one positive Lyapunov exponent. In Ref. 11 this phenomena was called "lag synchronization."'

\section{MAPPING APPROACH TO MULTISTABILITY}

To construct a model of the emergence of chaotic synchronization let us start by considering the following expression:

$$
x(t)=A(\phi(t)) \sin (\omega t) .
$$

Here, $\phi=\omega t$ is a phase of oscillations, and $A(\phi)$ $=\Pi_{i=1}^{N}$

$\left(1-\sigma_{i} \sin \left[\omega t / 2^{i}+i(\pi / 2)\right]\right)$ represents momentary amplitude, $\omega$ is the natural frequency of oscillation, $N$ defines the period of the considered signal $T=2^{N}(2 \pi / \omega)$, and $\sigma_{i}$ specifies the amplitude of each of the subharmonic components. The term
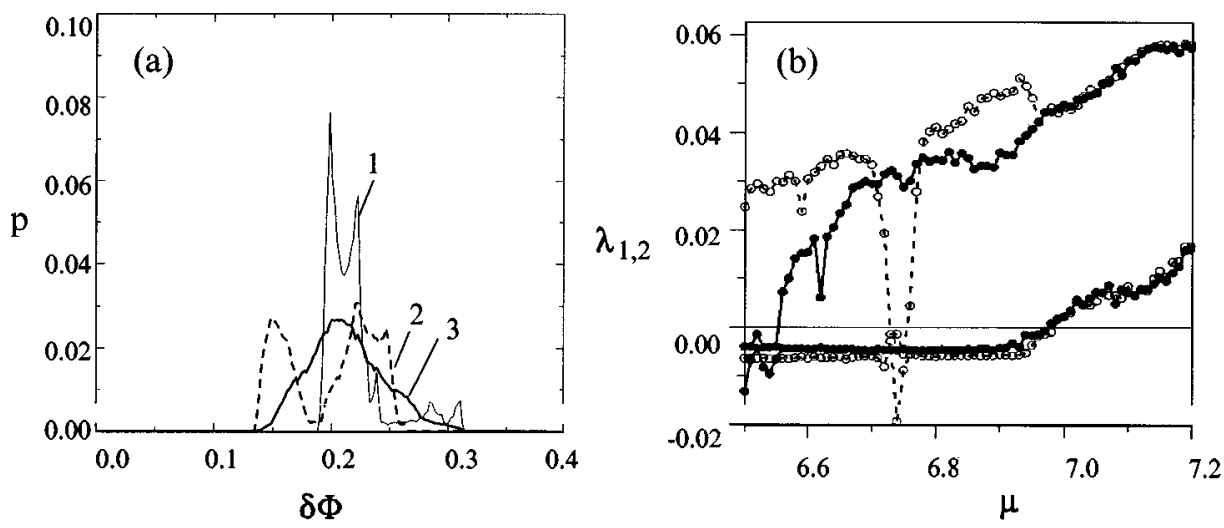

FIG. 3. Two coupled Rössler systems $(\alpha=0.15, \quad \beta=0.2, \quad c=0.02, \quad \Delta$ $=0.0093$ ): (a) The distribution of momentary phase differences for the synchronous chaotic attractors $C A_{0}$ and $C A_{1}$ (curve 1 and 2, respectively) at $\mu=6.6$ and for merged chaos $C A_{\Sigma}$ (curve 3) at $\mu=7.2$; (b) the largest Lyapunov exponents vs the parameter $\mu$ for the synchronous regimes of two families (dark points correspond to $C A_{0}$ and open circles to $C A_{1}$ ). 

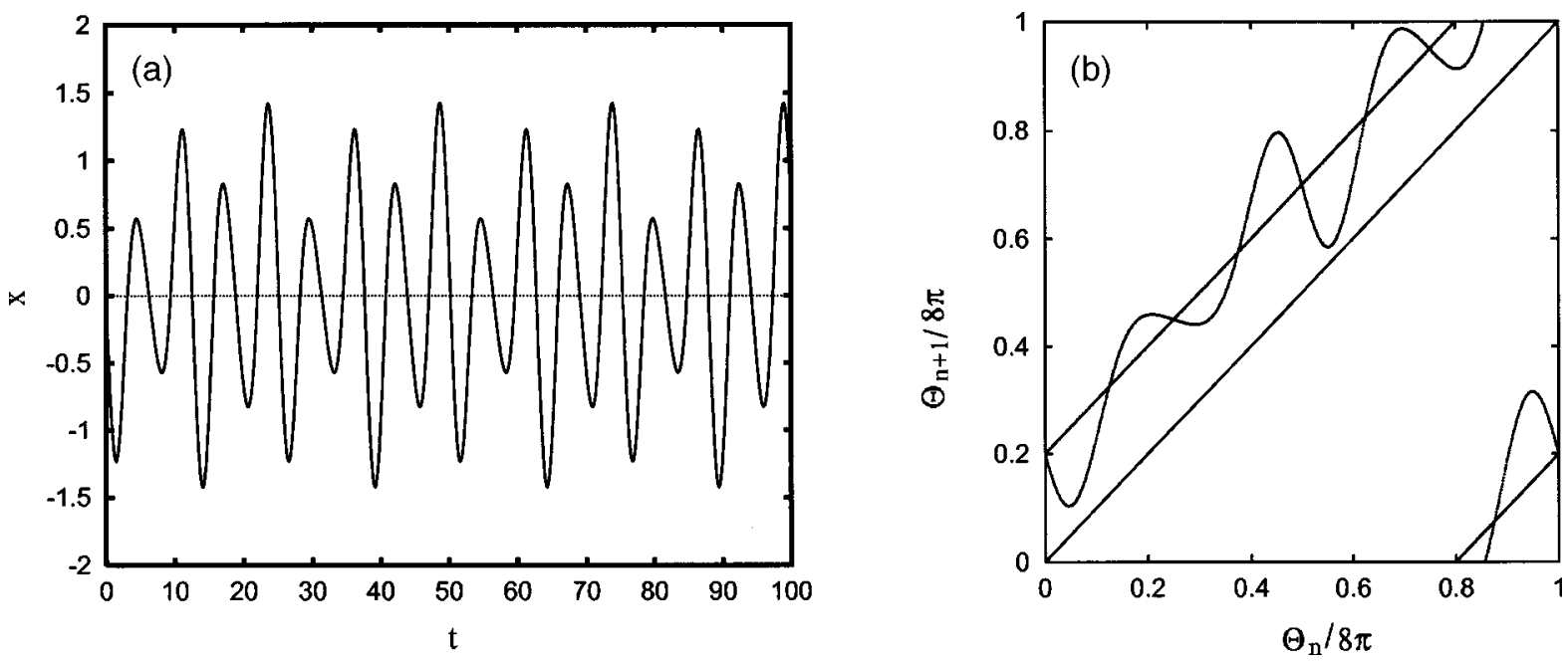

FIG. 4. (a) Time series $x(t)$ for the periodic orbits with period $4 T_{0}$ simulated from the expression (4). (b) The model map (10) for the case of $N=2$.

$i(\pi / 2)$ is introduced to obtain a more obvious phase portrait of each period-doubling in our model (in general, any phase shift may be used). The temporal variation described by (4) is illustrated in Fig. 4(a). As $N$ increases, $x(t)$ provides a qualitative representation of a sequence of high-periodic cycles leading in the limit to the birth of chaos via a cascade of period-doublings.

For two synchronized oscillators, each described by an expression like (4), the phase difference can attain $2^{N}$ different values, i.e., $\Theta=\phi_{1}-\phi_{2}=2 \pi m, \quad m=0,1,2, \ldots, 2^{N}-1$. Hence, the coexistence of a large number of periodic attractors will occur. When approaching the boundary of the synchronization region, these attractors disappear one by one, except for a single family whose bifurcations determine the transition to the nonsynchronous regime. In order to understand the structure of this boundary in more detail we shall investigate a sequence of model maps.

For quasiperiodic oscillators, the phase difference develops according to the following well-known equation: ${ }^{24}$

$$
\dot{\Theta}=\Delta-\gamma f\left(A_{1}, A_{2}\right) \sin \Theta .
$$

Here, $f(\cdot)$ is a function of the amplitudes $A_{1}$ and $A_{2}$ which is defined by the type of interaction, $\Delta$ represents the mismatch between the basic frequencies, and $\gamma$ is the coupling strength.

In our case the oscillators have different momentary phases $\phi_{1}$ and $\phi_{2}$ while their amplitudes depend on the phases in the following way:

$$
\begin{aligned}
& A_{1}=A\left(\phi_{1}\right)=\prod_{i=1}^{N}\left(1-\sigma_{i} \sin \left(\frac{\phi_{1}}{2^{i}}+i \frac{\pi}{2}\right)\right) \\
& A_{2}=A\left(\phi_{1}-\Theta\right)=\prod_{i=1}^{N}\left(1-\sigma_{i} \sin \left(\frac{\phi_{1}}{2^{i}}-\frac{\Theta}{2^{i}}+i \frac{\pi}{2}\right)\right) .
\end{aligned}
$$

It is not possible to obtain an explicit relation for the phase difference of two chaotic oscillators. However, qualitatively we can consider the oscillators as high-periodic cycles of periods $T=2^{N} 2 \pi / \omega$ where $\omega$ is the natural fre- quency of partial system $\left(\omega_{1}\right.$, for example). To use a discrete model, Eq. (5) should be integrated over the characteristic time $T$ of the system. Then we get

$$
\Theta_{n+1}^{N}=\Theta_{n}^{N}+\Omega-k F^{N}\left(\Theta_{n}^{N}\right) \bmod 2^{N} 2 \pi .
$$

Here, $\Theta_{n+1}^{N}=\Theta^{N}\left(t_{0}+n T\right)$ and $\Theta^{N} \in\left[0,2^{N} 2 \pi\right], \Omega=T \Delta$, and $K$ is a parameter which is related to the strength of interaction. It seems to be difficult to determine function $F^{N}$ analytically. We suppose that the intensity of interaction depends on the phase differences in the same way as the amplitude of subsystem vs its phase. As a simple approach we shall assume an expression of the form

$$
F^{N}\left(\Theta_{n}^{N}\right)=\sin \left(\Theta_{n}^{N}\right) \prod_{i=1}^{N}\left(1-\delta_{i} \sin \left(\frac{\Theta_{n}^{N}}{2^{i}}+i \frac{\pi}{2}\right)\right) .
$$

Equations (7) and (8) may be viewed as a generalization of the well-known circle map for simple oscillators. ${ }^{25}$ Varying $N=1,2,3, \ldots$, we obtain a family of maps, each being a model of synchronization for $2^{N}$-periodic cycles. The case of $N=2$ is illustrated in Fig. 4(b). The above equations are not normalized on the same scale because they are taken to the modulus $2^{N} 2 \pi$ which is changed with each period doubling. This allows us to preserve the values of $\Omega$ and $K$ and to compare the results for different $N$. A similar approach to construct a model map in the nonautonomous case was suggested by Pikovsky et al. ${ }^{26}$

With these preliminaries let us now investigate the structure of the boundary of the synchronization region for the main resonance $0: 1$ (or 1:1 for continuous-time systems). In terms of the map, the transition at that boundary corresponds to a tangent bifurcation. The condition for such a bifurcation to occur is

$$
\begin{aligned}
& \Theta_{*}^{N}+\Omega-K F^{N}\left(\Theta_{*}^{N}\right)=\Theta_{*}^{N}, \\
& \left.\frac{d\left(\Theta^{N}+\Omega-K F^{N}\left(\Theta^{N}\right)\right)}{d \Theta^{N}}\right|_{\Theta^{N}=\Theta_{*}^{N}}=1,
\end{aligned}
$$




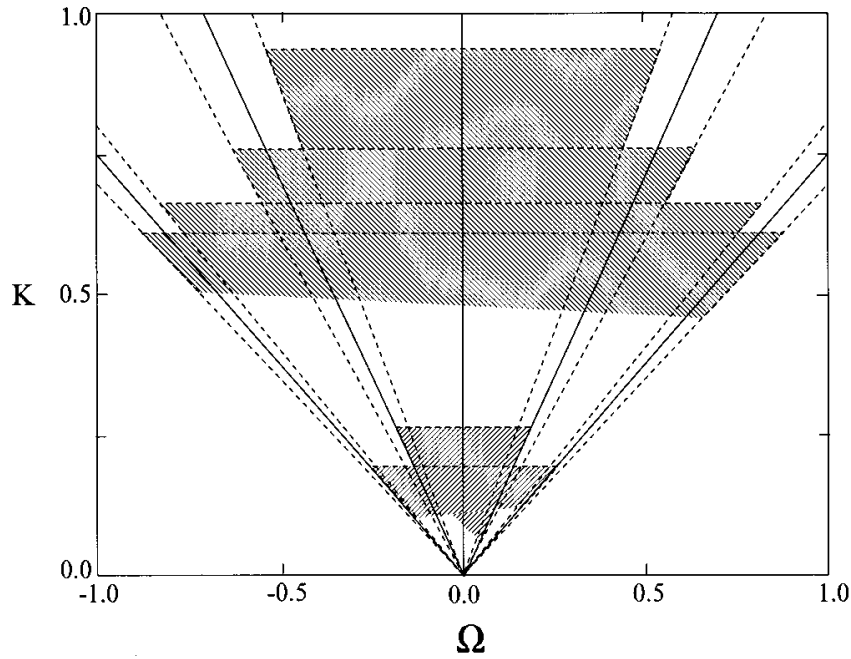

FIG. 5. Phase-locking regions for different families of attractors for (a) the model map (10) with $\delta=0.45$. The solid lines correspond to $N=1$ (two cycles of period-two coexist). The dashed lines correspond to $N=2$ (four cycles of period-four coexist).

where $\Theta_{*}^{N}$ is the fixed point. Equation (9) immediately gives

$$
\begin{aligned}
& K F^{N}\left(\Theta_{*}^{N}\right)=\Omega, \\
& \left.\frac{d F^{N}\left(\Theta^{N}\right)}{d \Theta^{N}}\right|_{\Theta^{N}=\Theta_{*}^{N}}=0 .
\end{aligned}
$$

Hence, it is easy to see that for any value of $\Theta_{*}^{N}$, the set of points corresponding to the tangent bifurcation forms a straight line in the $(\Omega, K)$ parameter plane. The number of roots of Eq. (10) defines the number of possible synchronous regimes. For the case of small $N$, Eq. (10) can be solved analytically. For larger $N$, this can be done numerically. Figure 5 shows the results for $N=1$ (fully drawn lines) and $N=2$ (dotted lines). Each line corresponds to the tangent bifurcation for one of the fixed points of the map. Under variation of $\Omega$, a pair of stable and unstable fixed points arises at each line. For larger $K$, the stable fixed point can subsequently lose its stability through a period-doubling bifurcation. To find the corresponding parameter values one only has to replace the zero on the right side of Eq. (10) by $2 / K$. However, in the present work we shall not consider the further bifurcations of the stable periodic solutions.

Thus, for small enough $K$ there are $2^{N}$ stable (and a similar number of unstable) fixed points near the center of the synchronization region. In terms of continuous-time dynamical systems, a set of stable fixed points corresponds to a set of possible synchronization regimes for the coupled oscillators. A two-dimensional torus exists both outside (where it is ergodic) and inside (where it is resonant) the synchronization region. Entering into the synchronization region corresponds to the birth of a pair of stable and saddle cycles, both lying on the torus surface. In these terms, the appearance and coexistence of other fixed points of the map represent the birth of additional pairs of stable and saddle cycles on the torus surface which do not intersect each other.

In this way we can draw the following conclusions concerning synchronization of high-periodic oscillations in

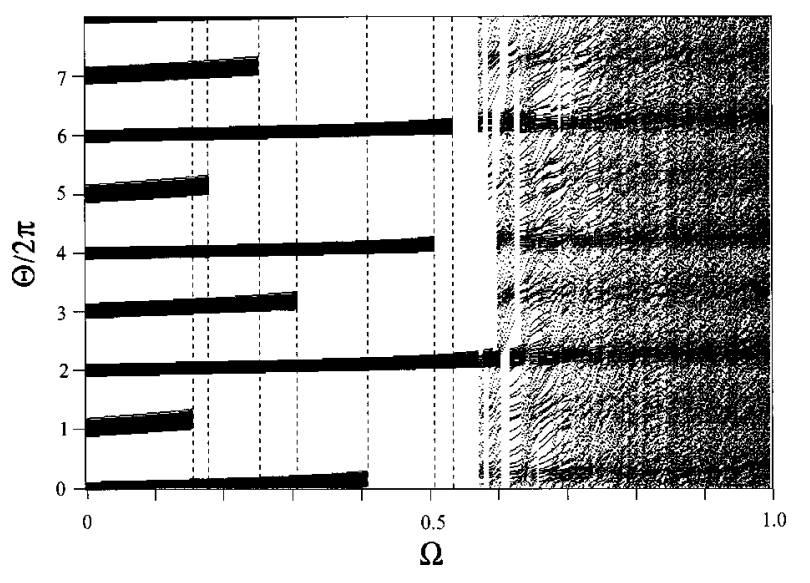

FIG. 6. One-parameter bifurcation diagram for the model map $(K=0.5$, $\sigma=0.45, B=1.2, N=3)$. The figure shows how the coexisting noise inflicted periodic orbits one by one lose their synchronization. Compare with Fig. 2, showing a similar phenomenon for the coupled Rössler systems.

coupled period-doubling systems: (i) There are $2^{N}$ coexisting synchronous solutions which differ from one another by phase shifts; and (ii) the boundary of synchronization for these solutions consists of a set of tongues inserted one into the other.

The question is now how the results listed here manifest themselves in the case of two interacting chaotic oscillators. We restrict our considerations to highly dissipative systems. Such systems can reasonably be characterized by a few specific time scales. The first of these is the return time to a surface of section (quasi-period of oscillations), and the second is the time constant characterizing the transient approach to some attractor. Thus, highly dissipative dynamical systems cannot distinguish an extremely high-periodic regime from a weakly-chaotic one if the envelops of their Fourier spectra are assumed to coincide. From another point of view, this type of chaotic motion may be considered as a regular behavior with an applied random excitation.

It is well-known that for the period-doubling route to chaos the chaotic attractor has an $N$-band structure $(N$ $=1,2,4, \ldots)$ within a range of control parameters. This structure is geometrically similar to the structure for the $\mathrm{N}$-periodic cycles. Thus, let us simulate an $\mathrm{N}$-band chaotic attractor using the model map (7) with an added noise term. The logistic map seems to be an appropriable source of such random forcing:

$$
\begin{aligned}
& \Theta_{n+1}^{N}=\Theta_{n}^{N}+\Omega-K F^{N}\left(\Theta_{n}^{N}\right)+B x_{n} \bmod 2^{N} 2 \pi, \\
& x_{n+1}=\lambda x_{n}\left(1-x_{n}\right),
\end{aligned}
$$

where the value of $\lambda$ is fixed at 3.99. Note that we introduce the source of noise in the above way (not the Gaussian noise, for example) to keep the multi-band structure of a chaotic attractor.

Within some range of noise amplitude $B$, the attractors produced by this equation become irregular but they still coexist in the phase space of the system and their basins of attraction differ. When $B$ is further increased, the merging of attractors becomes possible. ${ }^{27}$

Figure 6 shows a one-parameter bifurcation diagram for 
the case of an 8-band chaotic attractor. There are eight different synchronous chaotic regimes which coexist at small $\Omega$. When $\Omega$ increases the coexisting chaotic attractors one by one disappear at the edges of their respective synchronization regions. At $\Omega \geqslant 0.535$ a single synchronous solution is still stable. Note, how the "ghosts" of all eight synchronous solutions are still distinguishable inside the region of merged chaos at $\Omega>0.6$. The number of possible synchronous regimes decreases in the same way as for coupled Rössler systems (Fig. 2).

Hence, our conclusions with respect to synchronization of high-periodic regimes also apply for weakly-chaotic solutions. Moreover, we may conclude that (iii) the nested structure of synchronization tongues should be preserved in the case of an $N$-band chaotic attractor and remain similar to the structure for an $\mathrm{N}$-periodic cycle.

\section{CONCLUSIONS}

During the last few years a significant number of results have been obtained within the field of chaotic synchronization. However, many questions still remain open: What types of bifurcation can one observe at the transition from synchronous chaotic solutions to nonsynchronous solutions? What are the characteristic features of the synchronization of chaos with a different origin: chaos that appears via torus destruction, via intermittency, or via the merging of several attractors?

Based on a set of fairly general assumptions we have demonstrated the nested structure of phase synchronized regions for two dynamical systems with period-doublings and a weak diffusive coupling. These regions form the complex multisheet structure of the synchronization region for a set of coexisting synchronous solutions. Inside the region of chaotic oscillations multistability is extended up to the complete merging of multi-band chaotic attractors with various mean phase differences. The sequence of bifurcations associated with the merging of chaotic attractors from different families finally leads to the appearance of hyperchaos and to the destruction of phase locking.

We conclude our study by listing several important issues left for future investigation. First, the process of the loss of lag synchronization inside the region of phase synchronization would have to be studied. Second, phase-locking regions for each family of chaotic attractors should be investigated in terms of periodic orbits embedded in the chaos. Finally, the role of multistability in phase synchronization can be generalized to systems demonstrating other routes to chaos.

\section{ACKNOWLEDGMENTS}

This work is supported in part by RFFI (Grant No. 9801-16531). O.S. and E.M. acknowledge support from the Danish Research Academy.

${ }^{1}$ Y. Kuramoto, Chemical Oscillations, Waves and Turbulence (SpringerVerlag, Berlin, 1984).

${ }^{2}$ S. Strogatz, in Biomathematics (Springer, Berlin, 1993), Vol. 100.

${ }^{3}$ V. S. Anishchenko, T. E. Vadivasova, D. E. Postnov, and M. A. Safonova, Int. J. Bifurcation Chaos Appl. Sci. Eng. 2, 633 (1992).

${ }^{4}$ L. M. Pecora and T. L. Carroll, Phys. Rev. Lett. 64, 821 (1990).

${ }^{5}$ J. F. Heagy, T. L. Carroll, and L. M. Pecora, Phys. Rev. E 50, 1875 (1994).

${ }^{6}$ L. Kosarev and U. Parlitz, Phys. Rev. Lett. 74, 5028 (1995).

${ }^{7}$ V. Afraimovitch, N. Veritchev, and M. Rabinovitch, Izv. VUZ., Radiofiz. 29, 1050 (1986) (in Russian).

${ }^{8}$ N. F. Rulkov, M. M. Suschik, L. S. Tsimring, and H. D. I. Abarbanel, Phys. Rev. E 51, 980 (1995).

${ }^{9}$ L. Kosarev and U. Parlitz, Phys. Rev. Lett. 76, 1816 (1996).

${ }^{10}$ M. G. Rosenblum, A. S. Pikovsky, and J. Kurths, Phys. Rev. Lett. 76, 1804 (1996).

${ }^{11}$ M. G. Rosenblum, A. S. Pikovsky, and J. Kurths, Phys. Rev. Lett. 78, 4193 (1997).

${ }^{12}$ P. Cvitanović, Universality in Chaos (Adam Hilger, Bristol, 1984).

${ }^{13}$ C. Grebogi, E. Ott, and J. A. Yorke, Phys. Rev. Lett. 48, 1507 (1982).

${ }^{14}$ A. S. Pikovsky and P. Grassberger, J. Phys. A 24, 4587 (1991).

${ }^{15}$ Yu. L. Maistrenko, V. L. Maistrenko, A. Popovich, and E. Mosekilde, Phys. Rev. Lett. 80, 1638 (1998).

${ }^{16}$ V. S. Anishchenko, Pis'ma Zh. Tekh Fiz. 10, 629 (1984) [Sov. Tech. Phys. Lett. 10, 266 (1984)].

${ }^{17}$ V. S. Anishchenko, T. Kapitaniak, M. A. Safonova, and O. V. Sosnovtseva, Phys. Lett. A 192, 207 (1994).

${ }^{18}$ O. Sosnovtseva and E. Mosekilde, Int. J. Bifurcation Chaos Appl. Sci. Eng. 7, 1225 (1997).

${ }^{19}$ A. Pikovsky, G. Osipov, M. Rosenblum, M. Zaks, and J. Kurths, Phys. Rev. Lett. 79, 47 (1997).

${ }^{20}$ E. Posa, Jr., E. Ott, and M. H. Hess, Phys. Rev. Lett. 80, 1642 (1998).

${ }^{21}$ V. V. Astakhov, B. P. Bezruchko, E. N. Erastova, and E. P. Seleznev, J. Tekh. Fiz. 60, 19 (1990) [Sov. Phys. Tech. Phys. 35, 1122 (1990)].

${ }^{22}$ J. Rasmussen, E. Mosekilde, and C. Reick, Math. Comput. Simul. 40, 247 (1996).

${ }^{23}$ V. S. Anishchenko, V. V. Astakhov, T. E. Vadivasova, O. V. Sosnovtseva, and L. O. Chua, Int. J. Bifurcation Chaos Appl. Sci. Eng. 5, 1677 (1995).

${ }^{24}$ I. Blekhman, Synchronization in Science and Technology (ASME, New York, 1988).

${ }^{25}$ D. Rand, J. Sethna, S. Ostlund, and E. D. Siggia, Phys. Rev. Lett. 49, 132 (1982).

${ }^{26}$ A. Pikovsky, M. Zaks, M. Rosenblum, G. Osipov, and J. Kurths, Chaos 7, 680 (1997).

${ }^{27}$ J. P. Crutchfield, J. D. Farmer, and B. A. Huberman, Phys. Rep. 92, 45 (1982). 